\title{
Time Series Modeling of Surface EMG Based Hand Manipulation Identification via Expectation Maximization Algorithm
}

\author{
Yang Lu, Zhaojie Ju, Yurong Liu, Honghai Liu
}

\begin{abstract}
In this paper, we focus on the method of employing the expectation maximization (EM) algorithm to the modeling of surface electromyography (sEMG) signals based on hand manipulations via available time series of the measured data. The model for the sEMG is developed as a hidden Markov model (HMM) framework. In order to represent dynamical characteristics of sEMG when multichannel observation sequence are given, a stochastic dynamic process is included in it based on the maximum likelihood estimation (MLE) principle. By using the EM algorithm, the hidden model parameters and the feature of the signal can be identified easily. Ten people of different time series data sets of different hand grasps and in-hand manipulations captured from different subjects are collected. The two different classifiers were used to recognize these hand manipulation signal. Compared with time and time-frequency domains and their combination feature, the proposed algorithm of the inferred model gain better performance and demonstrate the effectiveness. The average identification accuracy rate is $93 \%$ and the maximum classification ratio is $100 \%$.
\end{abstract}

Keywords

Surface electromyography recognition; Hidden Markov model; Expectation maximization algorithm; Time series data.

\section{INTRODUCTION}

It is well known that there are existing strong relationship between hand manipulation and the movement of articulatory forearm muscles. Also, sEMG signal contains information sent from the human neural system to control the activation of muscles and the power of sEMG signal reflects the property of hand manipulation [13]. Because the sEMG signal is easy accessed and recorded from the remnant muscles of the stump, and in practical utilizing of multifunction upper limb prostheses is considered to be actual control signal, it has been under especially investigations in the past decade [17]. Although a lot of effort is being spent on improving these weaknesses, the efficient and effective method has yet to be developed.

Recently, a major concern in constructing a mathematical model of sEMG signal continues to improve the precision of the dynamical characteristics $[2,6,25]$. sEMG signal is indeed extensively used for human-machine

This work was supported in part by the National Natural Science Foundation of China under Grants 61374127 and 61422301 , the Natural Science Foundation of Heilongjiang Province of China under Grant F201428, the Scientific and Technology Research Foundation of Heilongjiang Education Department under Grants 12541061, the 12th Five-Year-Plan in Key Science and Technology Research of agricultural bureau in Heilongjiang province of China under Grant HNK125B-04-03, the Doctoral Scientific Research Foundation under Grant XDB2014-12 and the Foundation for Studying Abroad of Heilongjiang Bayi Agricultural University.

Y. Lu is with the College of Information Technology, Heilongjiang Bayi Agricultural University, Daqing 163319, P.R. China. (Email:luyanga@sina.com).

Y. Lu, Z. Ju and H. Liu are with the Intelligent System \& Biomedical Robotics Group, School of Computing, University of Portsmouth, Portsmouth PO1 3HE, U.K. (e-mail: zhaojie.ju@port.ac.uk; honghai.liu@port.ac.uk).

Y. Liu is with the Department of Mathematics, Yangzhou University, Yangzhou, Jiangsu 225002, P. R. China. He is also with the Communications Systems and Networks(CSN) Research Group, Faculty of Engineering, King Abdulaziz University, Jeddah 21589, Saudi Arabia. (Email:liuyurong@gmail.com). 
interfacing, for example for the control of prosthetic devices [4,27], by extracting significant feature of the signal for control. In [1,2], a novel recurrent neural network based on the hidden Markov model is used to establish the model of time series data in sEMG signal. In [10-12,24], similar techniques are used, but a hidden Markov model is derived from the physiological generation of the sEMG signal. In [6], fuzzy entropy is applied to measure the characterization of sEMG signal. In [25], nonlinear measures based on recurrence plot is exploited for evaluating the hidden dynamical characteristics of sEMG signal. In [21], an adaptive neuro-fuzzy inference system is proposed to identify hand motion commands and a hybrid algorithm is used to train and the average classification ratio is $92 \%$. In [14], The association of autoregressive models and a neural network is used for EMG pattern discrimination and the rates of success of control the movements of a virtual prosthesis is $100 \%$. In [8,9], a novel multiscale model and the iterated Hilbert transform is used for simulating the sEMG of an antagonistic pair of muscles during pathological tremor. In [22], the EMG signal processing of hand motion is represented by a linear multiple regression model. Advanced signal processing of the sEMG signal will inevitably promote the development of advanced multifunction upper limb prostheses, often called as "the mind-controlled artificial hands" [16].

This paper presents a new method to construct a model of time series data in sEMG signal. It differs from the existing research method in that we concentrate more on gaining deep insight into the relationship between the sEMG signal intensities and the hand manipulation movement control. In order to gain better control performance, the primary thing to do is to extract the main feature of the test sEMG signal. At the same time, a sEMG signal is assumed to be the output of a stochastic dynamic process driven by the physiological properties of the muscle and the form of the contraction [7,19]. Also, a hidden Markov model (HMM) is a representation of a type of random process, it can describe time-varying processes in signal processing. Accordingly, HMM model can be applied for representing the dynamic and stochastic character of sEMG signals. Therefore, the model output captures the sEMG signal's statistical properties according to some goodness measure.

In essence, in order to improve the accuracy for hand motions classification, the measured sEMG signal intensities can be described as a stochastic dynamic model, so the HMM stochastic dynamic process inevitably is contained into it. There are three main reasons why we select this model. Firstly in terms of the measured signal intensities, there is certain input-output causality. Secondly, in the course of observing measurement outputs of the model, there unavoidably exists noises owing to measurement accuracy. Finally since time series data that we got from digital computation is discrete, it is easy to analyse using the discrete-time stochastic dynamic model. Unfortunately, in previous research about sEMG model, few people pay attention on the issue of processing available time-series sEMG signals with certain noise.

In [35], the sEMG signal model identification (parameter estimation) is rather straightforward as it boils down to solving a system of linear equations. Different from this method, the focus of this paper is going to find a new way to jointly estimate the model parameters and the actual signal intensities simultaneously. The expectation maximization (EM) algorithm, firstly proposed by Shumway and Stoffer [32], is fit for dealing with the estimation problem via time series data depending on the above addressing features of the sEMG signal model. It can efficiently find maximum likelihood estimates of parameters in statistical model by using an iterative method. As we can see from [20,28-30,34,36-39], the EM algorithm has been widely used in all kinds of signal processing problems and gained computationally efficient estimations. The main motivation of studying the modeling method for time series data in sEMG signal is mainly twofold. 1) Up to now, it is still a challenge and is becoming the main focus of the on-going research in hand manipulation identification, 
in order to achieve a satisfactory rate for the sEMG signal recognition, the method of time-series modeling in sEMG signal is applied in this paper. 2) Based on the feature difference between sEMG signal of different hand manipulations, for continuous hand manipulation, HMM framework is used to extract and recognize sEMG signal feature.

As we all know, the sEMG signals recorded from natural behaviors are time-varying and nonstationary. For modeling these properties of the sEMG time series data, HMM framework has many advantages for some reasons. First, HMM framework is available for better representation of the dependent relationships of multivariate sEMG signals comes from different channels. Furthermore, HMM framework gives a probabilistically tractable and robust method of modeling the nonstationary and dynamic changes of state. On the basis of an analysis of the feature and parameters of HMM, the HMM framework can be applied to represent the feature of sEMG signal and related parameters. The main contribution of this paper is mainly twofold. 1) A HMM framework technique for modeling the sEMG signals is proposed to the issue of the hand manipulation recognition. Note that the proposed model can correctly and effectively recognize the hand manipulation. 2) Experiment results have shown that the EM algorithm that used for solving this model parameters can not only improve the convergence speed, but also obtain a higher recognition accuracy than other classical model such as AR, ARIMA, WAMP, RMS, MUAP and GMM.

The remaining of this paper is organized as follows. Section II describes a hidden Markov model for the sEMG signal. The EM algorithm is introduced in Section III for handling the parameter identification problem. Section IV applies EM algorithm to classify the collected data sets of sEMG and discussed the results. The last Section V gives related concluding remarks.

\section{Hidden Markov Model Framework For the SEMG Signal}

A sEMG signal represents an electrical signature of muscle activity [24]. It can be described by the stochastic dynamic model. Let $y_{i}(k) k \in[0, m]$ be a Markov chain taking values in a finite state space, in fact it is a sequence of observed signal, $S=\{1,2, \cdots, s\}, m$ is the measurement time points, $x_{i}(k) k \in[0, m]$ is actual value of the test sEMG signal, and $v_{i}(k) k \in[0, m]$ is an embedded measurement noise, suppose it is zero mean Gaussian white noise with covariance $V_{i}>0$. Thus, the sEMG signal model is in the following form:

$$
y_{i}(k)=H x_{i}(k)+v_{i}(k), \quad i=1,2, \cdots, n, \quad k=1,2, \cdots, m,
$$

where $H=\left[\begin{array}{ccc}x_{1}^{1} & \cdots & x_{1}^{n} \\ \cdots & \ddots & \vdots \\ x_{p}^{1} & \cdots & x_{p}^{n}\end{array}\right]$ is the design matrix with the time-series of a set of $p$ voxels. The shapes of the impulse $H$ represents the motor unit action potential shapes (MUAP) and are assumed time-variant. In this model, we do not consider of the effect of variable neuromuscular junction transmission and variable conduction velocities, it is a simplified representation of the actual sEMG signal.

Furthermore, we consider the following class of stochastic discrete-time system to model the sEMG signal containing $n$ states:

$$
\begin{aligned}
x_{i}(k+1) & =\sum_{j=1}^{n} H a_{i, j} x_{j}(k)+w_{i}(k), \\
i & =1,2, \cdots, n, \quad k=1,2, \cdots, m,
\end{aligned}
$$

where $a_{i, j}$ represents the relationship and degree amongst the value of test signal. $a_{i, j}>0$ means the $j$ th state positive stimulating the $i$ th state and, similarly, $a_{i, j}<0$ stands for the $j$ th state negative repressing the $i$ th 


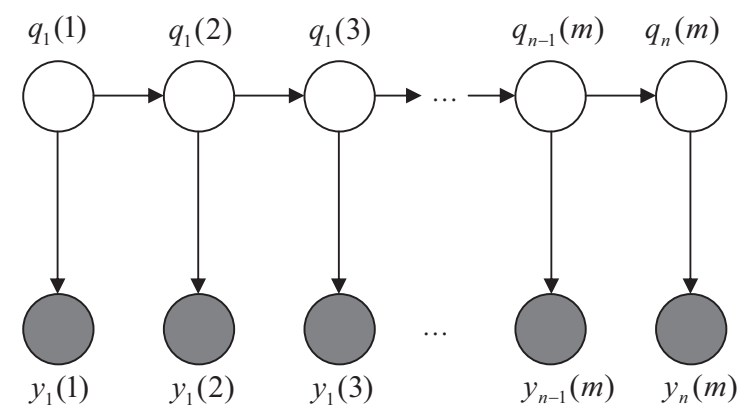

Fig. 1. The HMM model

state, while a value of zero indicates that $j$ th state does not influence the transcription of $i$ th state. $w_{i}(k)$ is the system noise. We also assume that $w_{i}(k)$ is a zero mean Gaussian white noise sequence with covariance $W_{i}>0$, and $w_{i}(k)$ and $v_{i}(k)$ are mutually independent.

Suppose

$$
x(k)=\left[\begin{array}{llll}
x_{1}(k) & x_{2}(k) & \cdots & x_{n}(k)
\end{array}\right]^{T}, k=1,2, \ldots, m
$$

and

$$
\alpha_{i}=\left[\begin{array}{llll}
\alpha_{i, 1} & \alpha_{i, 2} & \cdots & \alpha_{i, n}
\end{array}\right], i=1,2, \ldots, n
$$

It can be rewrite simplified as

$$
x_{i}(k+1)=H x(k)+w_{i}(k), \quad i=1,2, \cdots, n .
$$

In this paper, our aim is to establish the model (1) and (3) from the observed data

$$
\begin{array}{r}
Y:=\left\{y_{1}(1), y_{1}(2), \cdots, y_{1}(m), y_{2}(1), y_{2}(2), \cdots,\right. \\
\left.y_{2}(m), \cdots, y_{n}(1), y_{n}(2), \cdots, y_{n}(m)\right\} .
\end{array}
$$

Suppose that above sequence of observed data $y_{1}(1), y_{2}(2), \cdots, y_{n}(m)$, each of which is associated with a hidden state $q_{1}(1), q_{2}(2), \cdots, q_{n}(m)$, as shown in Fig. 1. In terms of the reference [26], we assume that the points are generated from an underlying density $p(x)$. Also, we further assume that $p(x)$ is defined as a finite hidden Markov model with $K$ components containing $n$ states:

$$
p(x \mid \Theta)=\sum_{k=1}^{n} \alpha_{k} p_{k}\left(x \mid z_{k}, \theta_{k}\right)
$$

where $p_{k}\left(x \mid z_{k}, \theta_{k}\right)$ is component, $1 \leq k \leq n$. Each is a density or distribution defined over $p(x)$ with parameters $\theta_{k} . z=\left(z_{1}, \cdots, z_{n}\right)$ is a vector, it is a stochastic variable that represents the identity of the component that generated $x$. The $\alpha_{k}=p\left(z_{k}\right)$ is the weights, representing the probability that randomly selected $x$, where $\sum_{k=1}^{n} \alpha_{k}=1$. Then the parameters for the proposed model with $n$ states is $\Theta=\left\{\alpha_{1}, \cdots, \alpha_{n}, \theta_{1}, \cdots, \theta_{n}\right\}$. Suppose we have observed data with noise, the function of maximum-likelihood estimation for noise observed data is: $L(\theta)=\sum_{i=1}^{n} \log \sum_{s_{1}, \cdots, s_{n}} p\left(x_{1}, \cdots, x_{n}, s_{1}, \cdots, s_{m} ; \theta\right)$, where $\theta \in \Theta$ is a $k \times 1$ parameter vector. 
Furthermore, suppose that we do not know the explicit form of $p(x \mid \Theta)$, instead we know there are some hidden variable $z$, and we can write down $p(x \mid \Theta)$ as an integration of the joint probability of $x$ and $z$, so $p(x \mid \Theta)=\log \sum_{x} p(z, x \mid \Theta)$. Directly maximizing $p(z, x \mid \Theta)$ of this form is difficult because the $\log \operatorname{term} \log \sum$ can not be further reduced. Instead of examining through all possible $x$ and maximizing their sum, we are going to use an iterative searching technique called Expectation-Maximization to maximize the log-likelihood and identify the model (1) and (3). The algorithm will converge to a local maximum of the likelihood function.

\section{An EM ALGORITHM FOR PARAMETER IDENTIFICATION}

In order to estimate the parameter of model (1) and (3), Expectation Maximization (EM) algorithm is used to learn the hidden parameters of aforementioned hidden Markov model, we make the following assumption:

Suppose that the joint probability $p(z, x \mid \Theta)$ falls into exponential families, we can write it down as, $p(z, x \mid \Theta)=\exp \{\langle g(\Theta, T(z, x))\rangle+d(\theta)+s(z, x)\}$. If the maximization likelihood estimate of $\theta$ exists, then it must be some function of the sufficient statistics $T(z, x) . \theta_{M L E}=\operatorname{argmax}_{\theta \in \Omega}\{\langle g(\Theta, T(z, x))\rangle+d(\theta)\}$, where $\Omega$ denotes the set of MLE estimate of $\theta$. So we can maximize this function using EM algorithm to solve this parameters identification problem.

Step One: Find a lower-bound of $\ell(\theta ; y)$ :

First we introduce a density function $q(x)$ called "averaging distribution". A lower-bound of the loglikelihood is given by, according to Jensen's inequality (log-concavity) [23].

$$
\begin{aligned}
\ell(\theta ; y) & =\log p(y \mid \theta) \\
& =\log \sum_{x} p(y \mid \theta) \\
& =\log \sum_{x} q(x) \frac{p(y, x \mid \theta)}{q(x)} \\
& \geq \sum_{x} q(x) \log \frac{p(x, y \mid \theta)}{q(x)} \\
& =E_{q(x)}[\log p(y, x \mid \theta)]+\operatorname{Entropy}[q(x)]
\end{aligned}
$$

The above equation $\ell(\theta ; y)$ can be decoupled as the sum of three terms:

$$
\begin{aligned}
\ell(\theta ; y)= & E_{q(x)}[\log p(y, x \mid \theta)]+K L[q(x) \| p(x \mid y, \theta)] \\
& +\operatorname{Entropy}[q(x)]
\end{aligned}
$$

Step Two: Maximize the bound over $\theta$ and $q(x)$ iteratively

From the equation 6 . The equality is reached only at $q(x)=p(x \mid y, \theta)$, and the entropy term is independent of $\theta$. So we have

E-step: $q^{t}=\operatorname{argmax}_{q} L\left(q, \theta^{t+1} ; y\right)=p\left(x \mid y, \theta^{t-1}\right)$

M-step:

$$
\begin{gathered}
\theta^{t}=\operatorname{argmax}_{\theta} L\left(q^{t}, \theta ; y\right) \\
=\operatorname{argmax}_{\theta} E_{q^{t}(x)}[\log p(y, x \mid \theta)] \\
Q\left(\theta^{\prime}, \theta\right)=E_{p\left(x \mid y, \theta^{\prime}\right)}[\operatorname{logp}(y, x \mid \theta)] \\
=E_{p\left(x \mid y, \theta^{\prime}\right)}[\langle g(\theta), T(y, x)\rangle+d(\theta)+s(y, x)] \\
=\left\langle g(\theta), E_{p\left(x \mid y, \theta^{\prime}\right)}[T(y, x)]\right\rangle+d(\theta)+\text { Constant }
\end{gathered}
$$


Let $\overline{T(y, x)}=E_{p\left(x \mid y, \theta^{\prime}\right)}[T(y, x)]$, the EM updating is then given by the recursion:

$$
\begin{aligned}
\theta_{E M}^{\prime \prime} & =\operatorname{argmax}_{\theta \in \Omega} Q\left(\theta^{\prime}, \theta\right) \\
& =\operatorname{argmax}_{\theta \in \Omega}\langle g(\theta), \overline{T(y, x)}\rangle+d(\theta)
\end{aligned}
$$

We conclude that when the complete data density is from exponential families, in the $M$ step the EM estimate of the parameters take the exactly same form as the MLE estimate.

The complete-data-log-likelihood is given by

$$
\begin{aligned}
\log p(q, y)= & \sum_{i=1}^{M} q_{0}^{i} \log \pi_{i}+\sum_{t=0}^{T} \sum_{j=1}^{M} q_{t}^{i} q_{t+1}^{j} \log \alpha_{i j} \\
& +\sum_{t=0}^{T} \sum_{j=1}^{M, N} q_{t}^{i} y_{t}^{j} \log \eta_{i j} \\
= & \sum_{i=1}^{M} q_{0}^{i} \log \pi_{i}+\sum_{i, j=1}^{M}\left(\sum_{t=0}^{T} q_{t}^{i} q_{t+1}^{j}\right) \log \alpha_{i j} \\
& +\sum_{i, j=1}^{M, N}\left(\sum_{t=0}^{T} q_{t}^{i} y_{t}^{j}\right) \log \eta_{i j}
\end{aligned}
$$

From the expression we see that the sufficient statistics for $\pi, \alpha, \eta$ are:

$$
q_{0}^{i} ; m_{i j}=\sum_{t=0}^{T} q_{t}^{i} q_{t+1}^{j} ; n_{i j}=\sum_{t=0}^{T} q_{t}^{i} y_{t}^{j}
$$

And they are subjective to the constraints:

$$
\sum_{i=1}^{M} \pi_{i}=1 ; \sum_{j=1}^{M} \alpha_{i}=1 ; \sum_{j=1}^{N} e t a_{i j}=1
$$

Applying Lagrange multiplier method, we obtain the MLE estimates of $\pi, \alpha$, and $\eta$,

$$
\begin{aligned}
\hat{\pi_{i}} & =q_{0}^{i} ; \\
\hat{\alpha_{i j}} & =\frac{m_{i j}}{\sum_{k=1}^{M} m_{i k}} ; \\
\hat{\eta_{i j}} & =\frac{m_{i j}}{\sum_{k=1}^{N} n_{i k}} ;
\end{aligned}
$$

So we get the EM estimates.

\section{Experimental Results and Discussions}

In this paper, we conduct four experiments by selecting different single feature and their combination based on in-hand manipulation of one person and multiple persons. We have known that traditional artificial neural network (ANN) is nonlinear model that is easy to use and understand, meanwhile back propagation (BP) learning algorithm is widely used in solving various classification and forecasting problems. Also, support vector machine (SVM) firstly developed by Vapnik in 1995 [33] is a supervised learning model with associated 


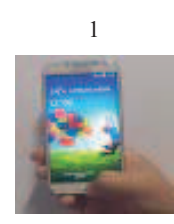

6

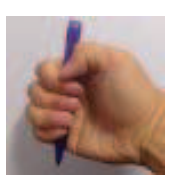

2

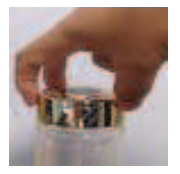

7

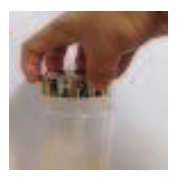

3

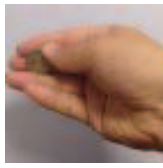

8

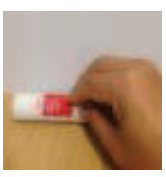

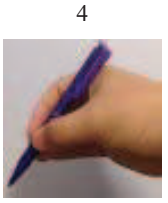

9

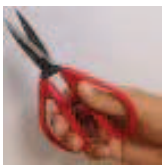

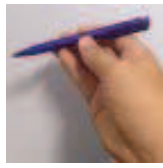

10

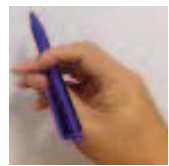

Fig. 2. Ten types of in-hand manipulation

learning algorithms that analyze data and recognize patterns, commonly used for classification and regression analysis [3]. So the SVM and BP neural network were applied to classify for classifying these 10 kinds of in-hand manipulation motions as classification.

The sEMG signal is recorded by 16 channels and there are 10 in-hand manipulations need to be identified. The number of neurons in the hidden layer had been tested between 16 and 10 neurons. We found that the best result of classification was achieved by 13 neurons. so we construct BP neural network structure to be 16-13-10, that is, there is 16 neural nodes in input layer, 13 neural nodes in hidden layer and 10 neural nodes in output layer. For the parameters of SVM, we use radial basis function as kernel function, penalty parameter $c$ and parameter $g$ were set to be 2 and 1 respectively according to the cross-validation method.

\section{A. Data Collection}

The time series data of sixteen channels of sEMG signals are collected from the Trigno Wireless Sensors sEMG capture system [17]. All the electrodes are placed on the forearm equidistantly and connecting to a hardware circuit through high quality wires. The monitoring software running on the computer can record the strength of corresponding sEMG signal.

In our experiment, we collect data from 10 participants including 3 women and 7 men. They are volunteered for the research and given informed consent prior to the experiments and the ethical approval for the study was obtained from University of Portsmouth CCI Faculty Ethics Committee. To separate each motion with next motions, before moving the hand, the hand should be the intermediate state which is a flat hand with no strength. In the course of data collection, each in-hand manipulations will be repeated to do 20 times by each person. The in-hand manipulations are shown in Fig. 2 and the motion described in Table I.

One collected raw sEMG signal of 16 channel and one separated channel are shown in Fig. 3 and Fig. 4 respectively.

\section{B. Pattern Recognition}

By using the proposed EM algorithm for extracting the features of the time series data in each channel, we can obtain samples to predict the hand manipulation.

According to [18], there are many classical sEMG feature representation method such as auto regressive model (AR), the autoregressive moving average (ARMA) model, integrated moving average (ARIMA), wavelet model and RMS represents root mean square and Wilson Amplitude (WAMP). In these models, RMS is one of the most popular and easy feature to represent EMG signal, WAMP represents Wilson Amplitude, it is an indicator of firing motor unit action potential (MUAP). AR is a prediction model that describe each sample 
TABLE I

DESCRIPTION OF TEN TYPES IN-HAND MANIPULATIONS

ID Description of in-hand manipulations

1 Open a mobile phone and then close it.

2 Screw to open a small bottle using only thumb,index finger and middle finger.

3 Pick up a coin and move it from the fingertip to the palm.

4 Remove the pencil from back of front for writing.

$5 \quad$ Pick up a pencil and simply rotate to write.

$6 \quad$ Pick up a pencil and complexly rotate to write.

7 Screw to open a big bottle using all five fingers.

8 Roll a small cylinder.

9 Pick up a scissor and cut paper.

10 Pencil flips.

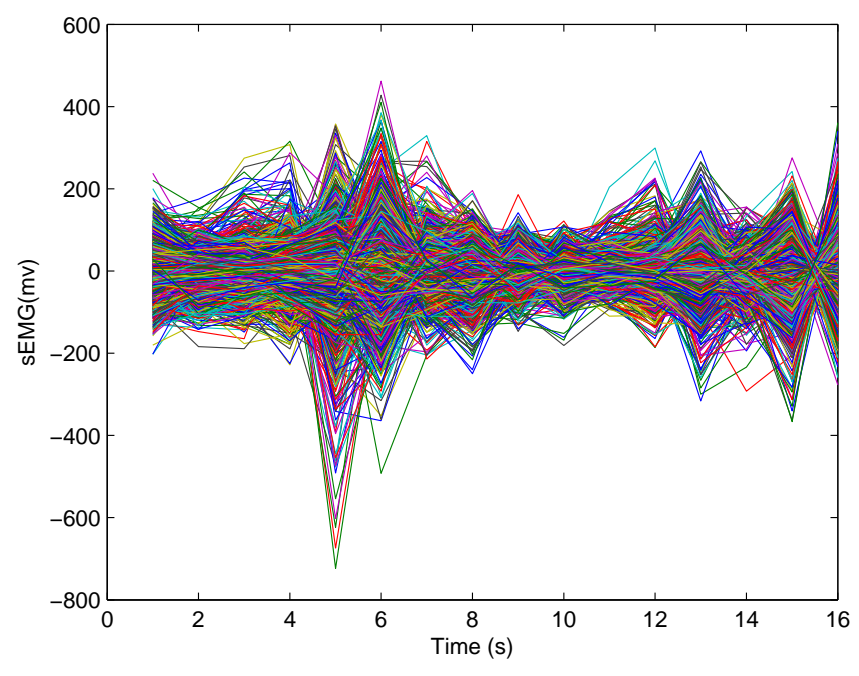

Fig. 3. The time series sEMG signal of 16 channels

of the EMG signal as a linear combination of the previous samples and a gaussian white noise error. Wavelet denotes the wavelet coefficients of sEMG signal. In pattern recognition problems, Gaussian Mixture Model (GMM) has been widely represented by a parametric probability density function.

For a better result display, we select the AR, ARMA, ARIMA, WAMP, RMS, MUAP and GMM model and their combination to compare with the proposed HMM model. The samples were divided into two parts, $50 \%$ for training and $50 \%$ for test. In order to get a mean accuracy, we use 10-fold cross validation method to processing the data. That is, we separate the data into 10 sets, 9 data sets using for training and the other one using for testing, then repeat 10 times.

\section{Identification With Single Feature and Single Person}

Firstly, the 10 in-hand manipulations samples of the sEMG signal of one person will be trained and tested based on single feature. The identification results are shown in Fig. 5. The HMM feature model has the best 


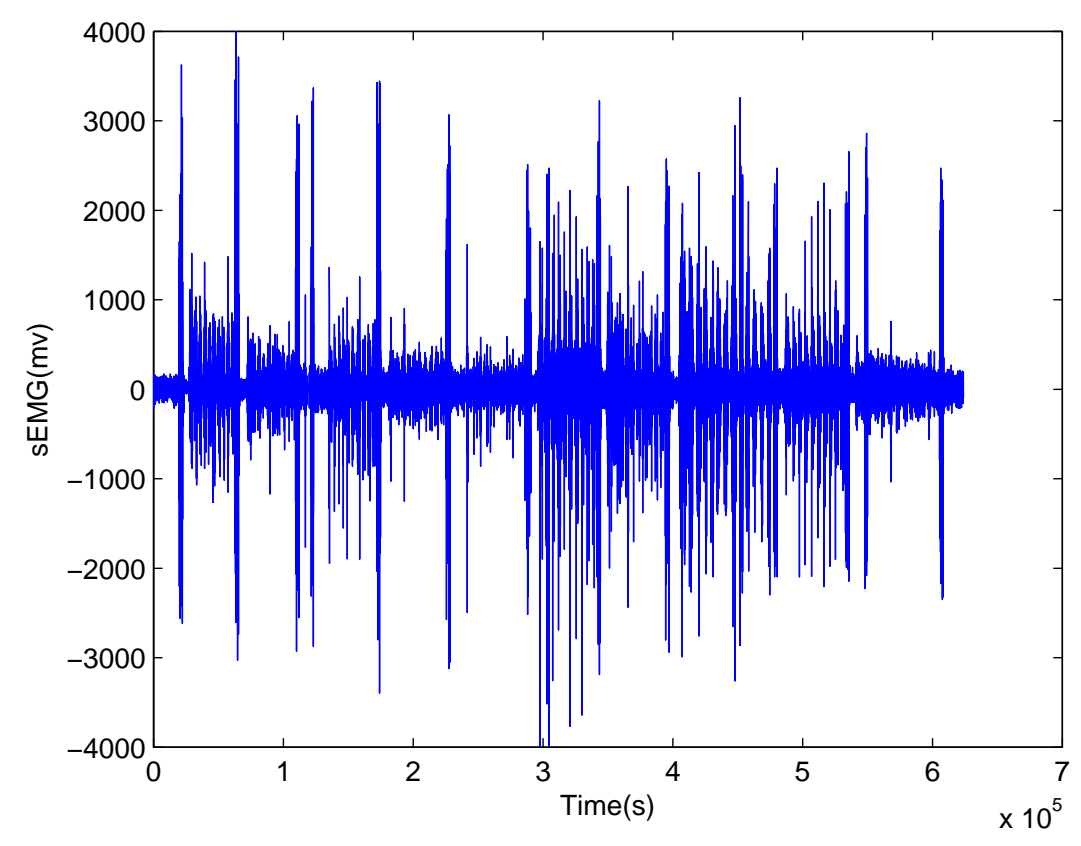

Fig. 4. The time series sEMG signal of 1 channels

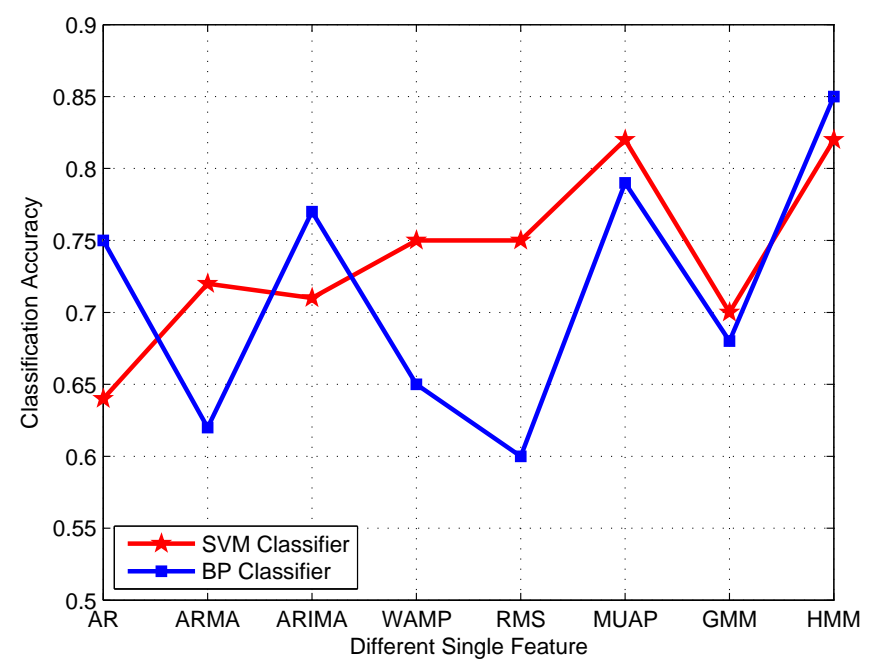

Fig. 5. Identification results based on different single feature

accuracy for any classification. The SVM classification performance is better than BP totally. But the highest identification rate for HMM and the lowest identification rate for RMS has been gained by BP. The other feature model have almost similar performance. Fig. 6 shows the confusion matrix for the ten different inhand manipulations using HMM feature. Manipulation 4 has the lowest rate of only $75 \%$, manipulation 5 have $100 \%$ accuracies, while manipulation 8 and manipulation 9 have $99 \%$. Secondly, we identify the 10 in-hand manipulations samples of the sEMG signal with single feature, but the data comes from multiple persons. Among 5 person's data used for training and other 5 person's data used for testing. The classification rate is lower than the first experiment. 


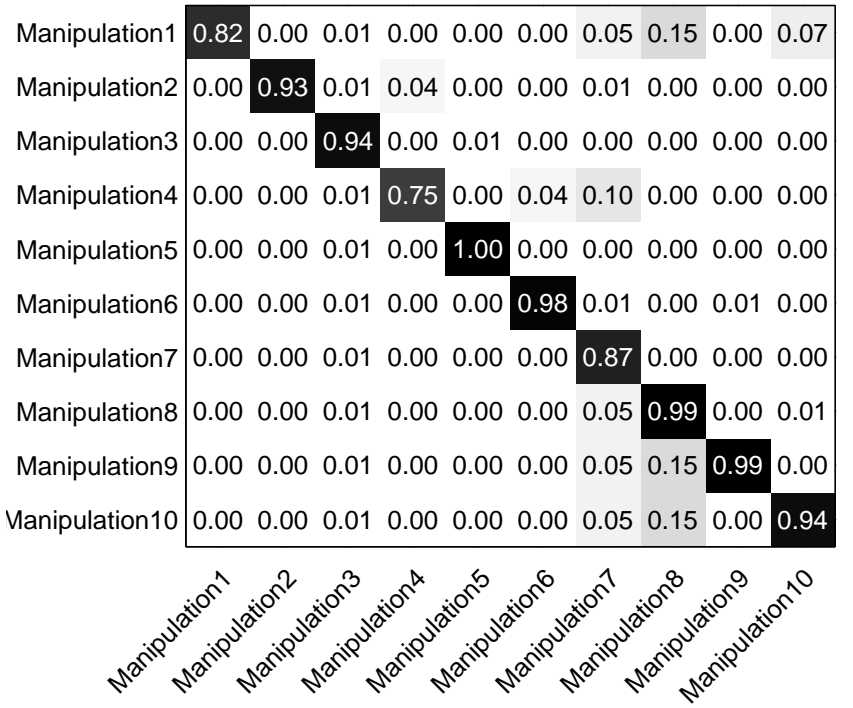

Fig. 6. Confusion matrix for the ten in-hand manipulations with HMM model

\section{Identification With Multiple Features and Single Person}

According to the reference $[17,22,25]$, the feature combination may has better performance than the single feature. The goal of the third experiment is used to show this kind of advantage of identification. The 10 in-hand manipulations samples of the sEMG signal of one person will be trained and tested based on feature combination. In Fig. 7, different feature combined with HMM respectively and the recognition results are compared with single HMM model. As we can see, multi-features have overall better and more stable performance than the single HMM model. Most of feature combination are above $80 \%$, and GMM+HMM gain the highest rate $95 \%$. Fig. 8 has shown the confusion matrix for the different in-hand manipulations by the GMM+HMM feature. Most of the motions have above $92 \%$ recognition rates, except the motion 4 has a $89 \%$ recognition rate. Furthermore, for the case of identification with multiple features and multiple persons, the recognition rate is lower than the third experiment.

\section{E. Average Identification Rate With Single and Multiple Features}

In order to get more precise identification result, 10 -fold cross validation was adopted to verify the above 4 experiments. The average recognition rate with single and multiple features are shown in Fig. 9 and Fig. 10 respectively. The classification accuracy of the in-hand manipulation is represented on $\mathrm{Y}$ axis. $\mathrm{X}$ axis represents different feature model and their combination. The experiment results have shown that HMM feature model has better performance than the other single feature, while the HMM model combined with other feature is better than the single HMM feature.

\section{Conclusion}

In this paper, we have applied the expectation maximization (EM) algorithm to model the sEMG signal via available time series of the measured signal intensities. The sEMG signal has been viewed as a Hidden Markov model. By using the EM algorithm, the model parameters, the actual signal intensities as well as the noise intensity have been identified simultaneously. Ten people hand grasps and in-hand manipulations 


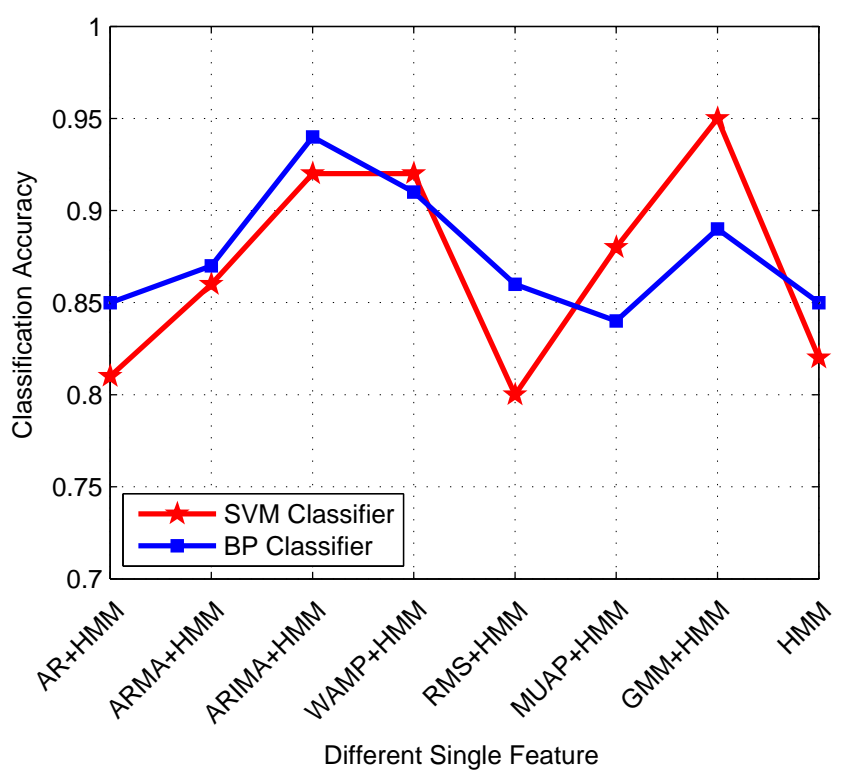

Fig. 7. Identification results based on different single feature combined with HMM model

of time series data sets concerning the prediction have been employed to demonstrate the effectiveness of the introduced algorithm. Several experiments results have also shown that the inferred model fits the data very well and gain better recognition rate. In near feature, we will continue to investigate the issue of improving hand manipulations recognition ratio via sEMG signal by applying better model or combined with other model. Besides EM algorithm, we will do more research on better algorithm to solve the model parameters. Furthermore, the knowledge base with hand manipulation can be applied into this framework automatically.

\section{REFERENCES}

[1] P. J. Brockwell and R. A. Davis, Time series: theory and methodsy, Springer, 2009.

[2] N. Bu, O. Fukuda and T. Tsuji, EMG-based motion discrimination using a novel recurrent neural network, Journal of Intelligent Information Systems, vol. 21, no. 2, pp. 113-126, 2003.

[3] C. Burges , A tutorial on support vector machines for pattern recognition, Data mining and knowledge discovery, Vol. 2, No. 2, pp. 121-167, 1998.

[4] C. Castellini, P. van der Smagt, G. Sandini and G. Hirzinger, Surface EMG for force control of mechanical hands, Proc. IEEE International Conference On Robitics and Automation, Pasadena, CA, USA, pp. 725-730, 2008.

[5] A.D.C. Chan and G.C. Green, Myoelectric cntrol development toolbox, Proc. 30th Conference of the Canadian Medical ES Biological Engineering Society, Toronto, CA, USA, 2007.

[6] W. Chen, Z. Wang, H. Xie and W. Yu, Characterization of surface EMG signal based on fuzzy entropy, IEEE Transactions on Neural Systems and Rehabilitation Engineering, vol. 15, no. 2, pp. 266-272, 2007.

[7] J. Chiang, Z.J. Wang and M.J. McKeown, A hidden markov, multivariate autoregressive (HMM-mAR) network framework for analysis of surface EMG data, IEEE Transactions on Biomedical Engineering, vol. 56, no. 8, pp. 4069-4081, 2008.

[8] J. L. Dideriksen, F. Gianfelici, L. Z. P. Maneski and D. Farina, EMG-based characterization of pathological tremor using the iterated Hilbert transform, IEEE Transactions on Biomedical Engineering, vol. 58, no. 10, pp. 2911-2921, 2011.

[9] J. L. Dideriksen, R. M. Enoka and D. Farina, A model of the surface electromyogram in pathological tremor, IEEE Transactions on Biomedical Engineering, vol. 58, no. 8, pp. 2178-2185, 2011.

[10] H. Dong, Z. Wang, D. W. C. Ho and H. Gao, Robust $H_{\infty}$ filtering for markovian jump systems with randomly occurring nonlinearities and sensor saturation: the finite-horizon case, IEEE Transactions on Signal Processing, Vol. 59, No. 7, pp. 30483057, 2011. 


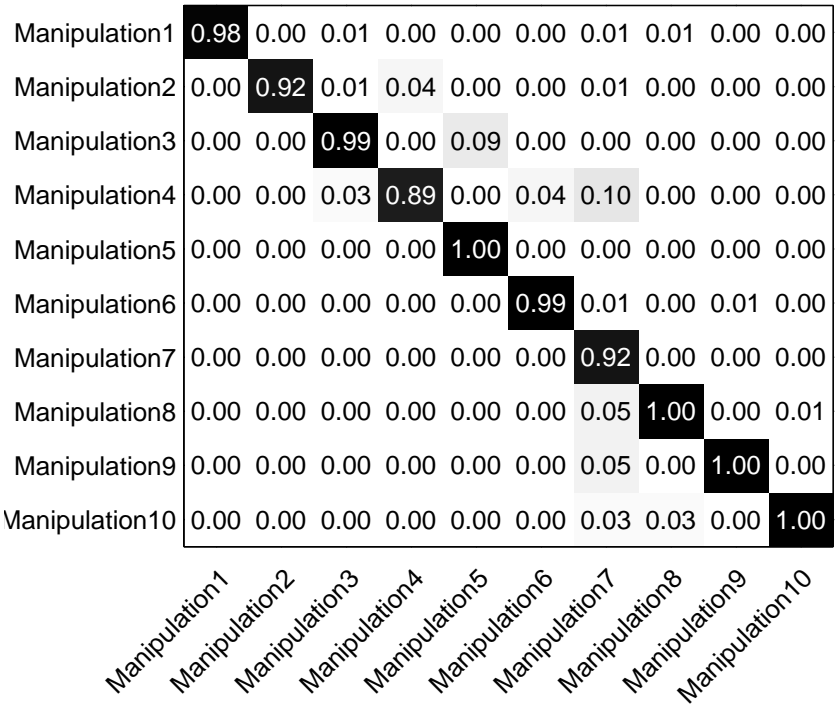

Fig. 8. Confusion Matrix for the ten in-hand manipulations with HMM combined with other model

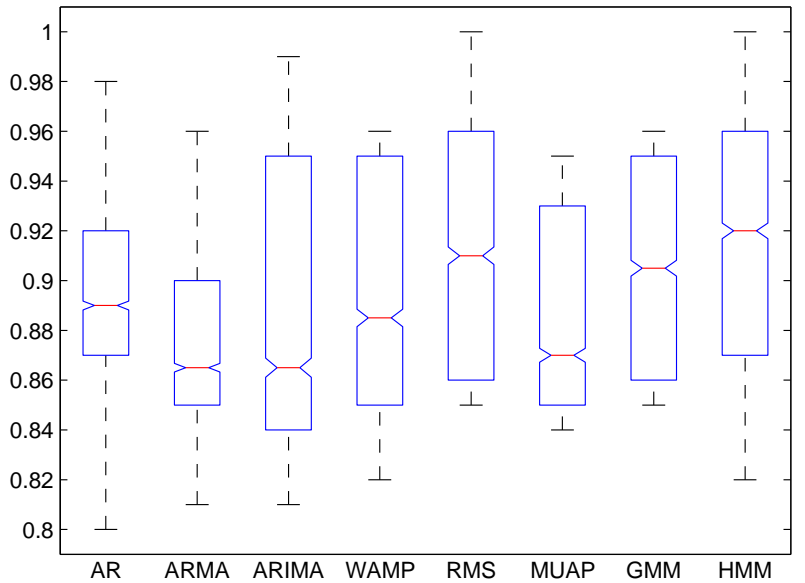

Fig. 9. Average recognition accuracy with single feature model

[11] H. Dong, Z. Wang and H. Gao, Distributed $H_{\infty}$ filtering for a class of Markovian jump nonlinear time-delay systems over lossy sensor networks, IEEE Trans. Industrial Electronics, Vol. 60, No. 10, pp. 4665-4672, 2013.

[12] H. Dong, Z. Wang and H. Gao, Fault detection for Markovian jump systems with sensor saturations and randomly varying nonlinearities, IEEE Trans. Circuits and Systems I: regular papers, Vol. 59, No. 10, pp. 23542362, 2012.

[13] H. Ghasemzadeh, R. Jafari and B. Prabhakaran, A body sensor network with electromyogram and inertial sensors: multimodal interpretation of muscular activities, IEEE Transactions on Information Technology in Biomedicine, vol. 14, no. 2, pp. 198206, 2010.

[14] D. Graupe and W. K. Cline, Functional separation of EMG signals via ARMA identification methods for prosthesis control purposes, IEEE Transactions on Systems, Man and Cybernetics, vol. 5, no. 2, pp. 252-259, 1975.

[15] G. Inoussa, H. Peng and J. Wu, Nonlinear time series modeling and prediction using functional weights wavelet neural network-based state-dependent AR model, Neurocomputing, vol. 86, no. 1/2, pp. 59-74, 2012.

[16] N. Jiang, S. Dosen, K.R. Mller and D. Farina, Myoelectric control of artificial limbsIs there a need to change focus, IEEE 


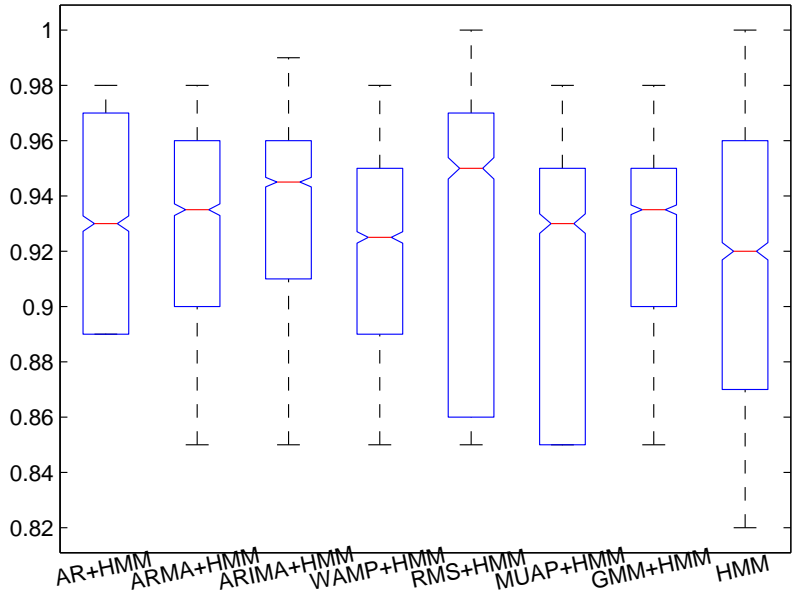

Fig. 10. Average recognition accuracy with multiple feature model

Signal Processing Magazine, vol. 29, no. 5, pp. 149-152, 2012.

[17] Z. Ju, G. Ouyang, M. Wilamowska-Korsak and H. Liu, Surface EMG Based Hand Manipulation Identification Via Nonlinear Feature Extraction and Classification, IEEE Sensors Journal, vol. 13, no. 9, pp. 3302-3311, 2013.

[18] B. Karlk, Machine Learning Algorithms for Characterization of EMG Signals, International Journal of Information and Electronics Engineering, Vol. 4, No. 3, pp. 189194, 2014.

[19] H. Kato, M. Taniguchi and M. Honda, Statistical analysis for multi-plicatively modulated nonlinear autoregressive model and its applications to electrophysiological signal analysis in humans, IEEE Transactions on Biomedical Engineering, vol. 54, no. 9, pp. 3414-3425, 2006.

[20] M.E. Khan, D.N. Dutt, An expectation-maximization algorithm based kalman smoother approach for event-related desynchronization (ERD) estimation from EEG, IEEE Transactions on Biomedical Engineering, vol. 54, no. 7, pp. 1191-1198, 2007.

[21] M. Khezri and M. Jahed, A neurofuzzy inference system for sEMG-based identification of hand motion commands, IEEE Transactions on Industrial Electronics, vol. 58, no. 5, pp. 1952-1960, 2011.

[22] T. Kitamura, N. Tsujiuchi and T. Koizumi, Hand motion estimation by EMG signals using linear multiple regression models, Proc. the 28th IEEE In Engineering in Medicine and Biology Society Annual International Conference, New York, USA, pp. 1339-1342, 2008.

[23] M. Kuczma, An introduction to the theory of functional equations and inequalities: Cauchy's equation and Jensen's inequality, Springer, 2008.

[24] J. Monsifrot, E. Le Carpentier, Y. Aoustin and D. Farina, Sequential Decoding of Intramuscular EMG Signals via Estimation of a Markov Model, IEEE Transactions on Neural Systems and Rehabilitation Engineering, vol. 22, no. 5, pp. 1030-1040, 2014 .

[25] G. Ouyang, X. Zhu, Z. Ju and H. Liu, Dynamical Characteristics of Surface EMG Signals of Hand Grasps via Recurrence Plot, IEEE Journal of Biomedical and Health Informatics, vol. 18, no. 1, pp. 257-265, 2014.

[26] L. Rabiner, A tutorial on hidden Markov models and selected applications in speech recognition, Proceedings of the IEEE , vol. 77 , no. 2, pp. 257-286, 1989.

[27] M. Reaz, M. Hussain and F. Mohd-Yasin, Techniques of EMG signal analysis: detection, processing, classification and applications, Biological Procedures Online, vol. 8, no. 1, pp. 11-35, 2006.

[28] Z. Wang, J. Eatock, S. McClean, D. Liu, X. Liu and T. Young. Modeling throughput of emergency departments via time series: an expectation maximization algorithm, ACM Transactions on Management Information Systems, vol. 4, no. 4, Art. No. 16, 2013.

[29] Z. Wang, F. Yang, D. W. C. Ho, S. Swift, A. Tucker and X. Liu, Stochastic dynamic modeling of short gene expression time series data, IEEE Transaction on NanoBioscience, vol. 7, no. 1, pp. 44-55, 2008. 
[30] Z. Wang, B. Zineddin, J. Liang, N. Zeng, Y. Li, M. Du, J. Cao and X. Liu. cDNA microarray adaptive segmentation, Neurocomputing, vol. 142, pp. 408-418, 2014.

[31] A. Soares, A. Andrade, E. Lamounier and R. Carrijo, The development of a virtual myoelectric prosthesis controlled by an EMG pattern recognition system based on neural networks, Journal of Intelligent Information Systems, vol. 21, no. 2, pp. 127-141, 2006 .

[32] R. H. Shumway and D. S. Stoffer, An approach to time series smoothing and forecasting using the EM algorithm, Journal of time series analysis, vol. 3, no. 4, pp. 253-264, 1982.

[33] V. N. Vapnik, The nature of statistical learning theory, New York: Springer, 1995.

[34] N. Zeng, Z. Wang, Y. Li, M. Du, J. Cao and X. Liu, Time series modeling of nano-gold immunochromatographic assay via expectation maximization algorithm, IEEE Transactions on Biomedical Engineering, vol. 60, no. 12, pp. 3418-3424, 2013.

[35] M. Zivanovic, Time-Varying Multicomponent Signal Modeling for Analysis of Surface EMG Data, IEEE Signal Processing Letter, vol. 21, no. 6, pp. 692-696, 2014.

[36] Singh K. B. and Taheri S., Estimation of tireroad friction coefficient and its application in chassis control systems, Systems Science 8 Control Engineering: An Open Access Journal, vol. 3, no. 1, pp. 39-61, 2015.

[37] N. Zeng, Z. Wang, Y. Li, M. Du and X. Liu, Time series modeling of nano-gold immunochromatographic assay via expectation maximization algorithm, IEEE Transactions on Nanotechnology, vol. 11, no. 2, pp. 321-327, 2012.

[38] D. Ding, Z. Wang, B. Shen and H. Dong, Envelope-constrained $H_{\infty}$ filtering with fading measurements and randomly occurring nonlinearities: The finite horizon case, Automatica, vol. 55, pp. 37-45, 2015.

[39] D. Ding, Z. Wang, J. Lam and B. Shen, Finite-horizon $H_{\infty}$ control for discrete time-varying systems with randomly occurring nonlinearities and fading measurements, IEEE Transactions on Automatic Control, in press, DOI: 10.1109/TAC.2014.2380671. 